\title{
Limiters for Unstructured Higher-Order Accurate Solutions of the Euler Equations
}

\author{
Krzysztof Michalak* and Carl Ollivier-Gooch ${ }^{\dagger}$ \\ Advanced Numerical Simulation Laboratory \\ University of British Columbia
}

\begin{abstract}
Higher-order finite-volume methods have been shown to be more efficient than secondorder methods. However no consensus has been reached on how to eliminating the oscillations caused by solution discontinuities. Essentially non-oscillatory (ENO) schemes provide a solution but are computationally expensive to implement and may not converge well for steady-state problems. This work studies the application of limiters used for second-order methods to the higher-order case. Requirements for accuracy and efficient convergence are discussed. A new limiting procedure is proposed. Results for the fourth-order accurate solution of transonic and supersonic flows demonstrate good convergence properties and significant qualitative improvement of the solution relative the second-order method. Subsonic results demonstrate the superiority of the scheme in smooth flows by the reduction in entropy production. Some aspects of the new limiter can also be successfully applied to reduce the dissipation of second-order schemes with minimal sacrifices in convergence properties.
\end{abstract}

\section{Introduction}

Higher-order discretizations have been shown to reduce computational effort on structured grids. ${ }^{1,2}$ Higher-order finite-volume methods on unstructured grids, although well known, ${ }^{3-5}$ have not effectively been applied to large scale aerodynamics problems. An outstanding issue with these methods is how to deal with discontinuities, such as shocks in the flow, while maintaining good accuracy and convergence.

One means of dealing with discontinuities is to use the classic MUSCL ${ }^{6}$ scheme with the addition of a slope limiter. For the second-order case, Barth and Jespersen ${ }^{7}$ demonstrated the use of limited-reconstruction for the solution of the Euler equations. Efficient convergence to steady-state was achieved by Venkatakrishnan ${ }^{8}$ by modifying the limiter to be differentiable.

Third order accurate schemes using $k$-exact reconstruction have been demonstrated first by Barth and Frederickson ${ }^{3}$ and subsequently by others $[5,9-12$, for example]. Transonic and supersonic solutions have been computed in some of these works by various extensions of second-order limiters. However, the work of Barth ${ }^{9}$ presents a limiting approach which causes difficulties in steady-state convergence, while other works ${ }^{5,12}$ present approaches that do not strictly enforce monotonicity and therefore allow some undesirable oscillations to occur.

An alternative to MUSCL for obtaining higher-order accurate solutions is the essentially non-oscillatory (ENO) scheme [13-15, for instance]. These methods avoid the need for slope limiters by selecting a smooth flux stencil at each iteration. Due to the inherent non-differentiability of this process, convergence of the solution to steady-state is not possible. Weighed ENO (WENO) [16-18, for instance] schemes were introduced in part to resolve this issue. However the convergence properties of these schemes continue to underperform those of MUSCL schemes. The computational cost per residual evaluation is also much higher than for reconstruction based solvers. A hybrid between WENO and MUSCL schemes named Quasi-ENO ${ }^{19}$ has similar limitations. This method's reconstruction step is much more expensive than traditional MUSCL since the reconstruction least-squares matrix changes at each iteration.

*PhD student, Student member AIAA. michalak@mech.ubc.ca

$\dagger$ Associate Professor, Member AIAA. cfog@mech.ubc.ca 
The present work aims to formulate the requirements and present a candidate for a limiter that achieves fourth-order accurate solutions in smooth regions while maintaining good convergence properties. An overview of the high-order MUSCL scheme is given in Section II. Second order-limiters are reviewed in Section III. Our extension of these methods to higher-order schemes is presented in Section IV. Finally, results for subsonic, transonic and supersonic flows are analyzed in Section V.

\section{Higher-Order Solution Reconstruction}

The third- and fourth-order accurate reconstruction procedure we use here is documented by OllivierGooch and Van Altena ${ }^{20}$ and is briefly reviewed in this section. Only the equations that are needed for the discussion of limiters are presented.

In the finite-volume method, the domain is tessellated into non-overlapping control volumes. Each control volume $V_{i}$ has a geometric reference point $\vec{x}_{i}$. While in principle any point can be chosen as the reference point, the usual choices (which we recommend) are the cell centroid for cell-centered control volumes and the vertex for vertex-centered control volumes. For any smooth function $u(\vec{x})$ and its control-volume averaged values $\bar{u}_{i}$, the $k$-exact least-squares reconstruction will use a compact stencil in the neighborhood of control volume $i$ to compute an expansion $R_{i}\left(\vec{x}-\vec{x}_{i}\right)$ that conserves the mean in control volume $i$ and reconstructs exactly polynomials of degree $\leq k$ (equivalently, $R_{i}\left(\vec{x}-\vec{x}_{i}\right)-u(\vec{x})=\mathcal{O}\left(\Delta x^{k+1}\right)$ ).

Conservation of the mean requires that the average of the reconstructed function $R_{i}$ and the original function $u$ over control volume $i$ be the same:

$$
\frac{1}{V_{i}} \int_{V_{i}} R_{i}\left(\vec{x}-\vec{x}_{i}\right) d A=\frac{1}{V_{i}} \int_{V_{i}} u(\vec{x}) d A \equiv \bar{u}_{i} .
$$

The expansion $R_{i}\left(\vec{x}-\vec{x}_{i}\right)$ can be written as:

$$
\begin{aligned}
R_{i}\left(\vec{x}-\vec{x}_{i}\right)= & \left.u\right|_{\vec{x}_{i}}+\left.\frac{\partial u}{\partial x}\right|_{\vec{x}_{i}}\left(x-x_{i}\right)+\left.\frac{\partial u}{\partial y}\right|_{\vec{x}_{i}}\left(y-y_{i}\right) \\
& +\left.\frac{\partial^{2} u}{\partial x^{2}}\right|_{\vec{x}_{i}} \frac{\left(x-x_{i}\right)^{2}}{2}+\left.\frac{\partial^{2} u}{\partial x \partial y}\right|_{\vec{x}_{i}}\left(\left(x-x_{i}\right)\left(y-y_{i}\right)\right) \\
& +\left.\frac{\partial^{2} u}{\partial y^{2}}\right|_{\vec{x}_{i}} \frac{\left(y-y_{i}\right)^{2}}{2}+\cdots
\end{aligned}
$$

Taking the control volume average of this expansion over control volume $i$ and equating it to the mean value gives

$$
\begin{aligned}
\bar{u}_{i}= & \left.u\right|_{\vec{x}_{i}}+\left.\frac{\partial u}{\partial x}\right|_{\vec{x}_{i}} \bar{x}_{i}+\left.\frac{\partial u}{\partial y}\right|_{\vec{x}_{i}} \bar{y}_{i} \\
& +\left.\frac{\partial^{2} u}{\partial x^{2}}\right|_{\vec{x}_{i}} \frac{\overline{x^{2}}}{2}+\left.\frac{\partial^{2} u}{\partial x \partial y}\right|_{\vec{x}_{i}} \overline{x y}+\left.\frac{\partial^{2} u}{\partial y^{2}}\right|_{\vec{x}_{i}} \frac{\overline{y^{2}}}{2}+\cdots
\end{aligned}
$$

where

$$
\bar{x}^{n} y^{m}{ }_{i} \equiv \frac{1}{A_{i}} \int_{V_{i}}\left(x-x_{i}\right)^{n}\left(y-y_{i}\right)^{m} d A .
$$

are control volume moments. This condition, which must be satisfied exactly, is combined with the reconstruction goal of approximating nearby control-volume averages to obtain a constrained least-squares problem for the solution of the Taylor series expansion coefficients. Since the resulting least-squares matrix depends only on geometric terms, its pseudo-inverse may be found in a prepossessing step. Therefore the reconstruction step at each flux evaluation is reduced to a matrix-vector product and the exact flux Jacobian can be computed. ${ }^{21}$ Ollivier-Gooch ${ }^{19}$ presents a modification to the reconstruction procedure resulting in a quasi-ENO scheme. This scheme eliminates the requirement for a limiter by varying the weights of the rows in the least-squares matrix at each iterations based on a measure of smoothness. However, since the pseudo-inverse can no longer be precomputed, this scheme is computationally expensive. 


\section{Second Order Limiting}

A sufficient condition to avoid introducing oscillation in the solution process is that no new local extrema are formed during reconstruction. Barth and Jespersen ${ }^{7}$ introduced the first limiter for unstructured grids. The scheme consists of finding a value $\Phi_{i}$ in each control-volume that will limit the gradient in the piecewiselinear reconstruction of the solution. In the second-order reconstruction case, if the reference location $\vec{x}_{i}$ is taken to be the control-volume centroid, the point-wise value $\left.u\right|_{\vec{x}_{i}}$ is equal to the control volume average $\bar{u}_{i}$. This leads to the limited reconstruction of the form

$$
R_{i}\left(\vec{x}-\vec{x}_{i}\right)=\bar{u}_{i}+\Phi_{i} \nabla u_{i} \cdot\left(\vec{x}-\vec{x}_{i}\right), \Phi \in[0,1]
$$

The goal is to find the largest $\Phi_{i}$ which prevents the formation of local extrema at the flux integration Gauss points. The following procedure is used by Barth and Jespersen :

1. Find the largest negative $\left(\delta u_{i}^{\min }=\min \left(\bar{u}-\bar{u}_{i}\right)\right)$ and positive $\left(\delta u_{i}^{\max }=\max \left(\bar{u}-\bar{u}_{i}\right)\right)$ difference between the solution in the immediate neighbors and the current control volume.

2. Compute the unconstrained reconstructed value at each Gauss point $\left(u_{i j}=R_{i}\left(\vec{x}_{j}-\vec{x}_{i}\right)\right)$.

3. Compute a maximum allowable value of $\Phi_{i j}$ for each Gauss point $j$.

$$
\Phi_{i j}=\left\{\begin{array}{cl}
\min \left(1, \frac{\delta u_{i}^{\max }}{u_{i j}-\bar{u}_{i}}\right), & \text { if } u_{i j}-\bar{u}_{i}>0 \\
\min \left(1, \frac{\delta u_{i}^{\text {min }}}{u_{i j}-\bar{u}_{i}}\right), & \text { if } u_{i j}-\bar{u}_{i}<0 \\
1, & \text { if } u_{i j}-\bar{u}_{i}=0
\end{array}\right.
$$

4. Select $\Phi_{i}=\min \left(\Phi_{i j}\right)$.

Clearly, steps 1, 3, and 4 introduce non-differentiability in the computation of the reconstructed function. Consequently, the second-order flux is also non-differentiable. This adversely affects the convergence properties of the solver. In practice, the non-differentiability of step 3 causes the greatest degradation in convergence performance.

For this reason, Venkatakrishnan ${ }^{8}$ introduces a smooth alternative to step 3 of the Barth-Jespersen procedure by replacing the function $\min (1, y)$ with

$$
\phi(y)=\frac{y^{2}+2 y}{y^{2}+y+2}
$$

The effect of this modification can be seen in Figure 1. Although this new function is differentiable, it has

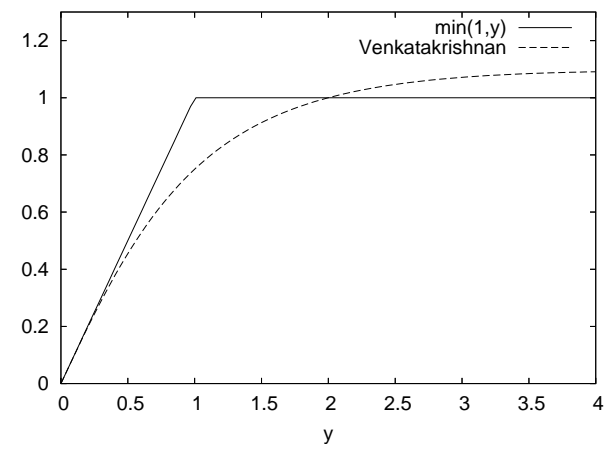

Figure 1. Venkatakrishnan's smooth approximation to $\min (1, y)$.

the disadvantage of reducing the gradient even in regions where no new extrema are formed. However, for smooth solutions on a uniform mesh, the value of the $\frac{\delta u_{i}^{\max }}{u_{i j}-\bar{u}_{i}}$ is expected to be $2 \pm \mathcal{O}\left(x_{i}-x_{i j}\right)$, and $\phi(y)$ is therefore expected to be $1 \pm \mathcal{O}\left(x_{i}-x_{i j}\right)$. Hence, modifying the gradient in this manner for second-order solutions introduces an error in the reconstruction which is on the order of truncation for smooth flows. 
A further modification introduced by Venkatakrishnan ${ }^{8}$ is a method to avoid applying the limiter in regions of nearly uniform flow. By eliminating the effect of the limiter when $u_{i j}-\bar{u}_{i} \leq(K \Delta x)^{1.5}$, where $\Delta x$ is a characteristic length for the control-volume and $K$ is a tunable parameter, accuracy and convergence can be improved.

For the case $u_{i j}-\bar{u}_{i}>0$ the limiter becomes

$$
\Phi_{i j}=\frac{1}{\Delta_{-}}\left[\frac{\left(\Delta_{+}^{2}+\epsilon^{2}\right) \Delta_{-}+2 \Delta_{-}^{2} \Delta_{+}}{\Delta_{+}^{2}+2 \Delta_{-}^{2}+\Delta_{-} \Delta_{+}+\epsilon^{2}}\right]
$$

In this equation $\Delta_{-}=u_{i j}-\bar{u}_{i}, \Delta_{+}=\delta u_{i}^{\max }$ and $\epsilon^{2}=(K \Delta x)^{3}$. With $K>0$, this modification does not maintain strict monotonicity.

\section{Higher Order Limiting}

\section{IV.A. Monotonicity}

The first challenge of extending the limiting procedure to third- and fourth-order accurate schemes is to express the monotonicity requirement including the higher-order reconstruction terms. Equation 2 can be rewritten in terms of the control-volume average with the help of equation 3 to yield:

$$
\begin{aligned}
R_{i}\left(\vec{x}-\vec{x}_{i}\right)= & \bar{u}_{i}+\left(\left.\frac{\partial u}{\partial x}\right|_{\vec{x}_{i}}\left(\left(x-x_{i}\right)-\bar{x}_{i}\right)+\left.\frac{\partial u}{\partial y}\right|_{\vec{x}_{i}}\left(\left(y-y_{i}\right)-\bar{y}_{i}\right)\right. \\
& +\left.\frac{\partial^{2} u}{\partial x^{2}}\right|_{\vec{x}_{i}}\left(\frac{\left(x-x_{i}\right)^{2}}{2}-\frac{\overline{x^{2}}}{2}\right)+\left.\frac{\partial^{2} u}{\partial x \partial y}\right|_{\vec{x}_{i}}\left(\left(x-x_{i}\right)\left(y-y_{i}\right)-\overline{x y}\right) \\
& \left.+\left.\frac{\partial^{2} u}{\partial y^{2}}\right|_{\vec{x}_{i}}\left(\frac{\left(y-y_{i}\right)^{2}}{2}-\frac{\overline{y^{2}}}{2}\right)+\cdots\right)
\end{aligned}
$$

This can be interpreted as meaning that the reconstructed solution at any point is the control-volume average plus second and higher-order contributions from the reconstruction :

$$
R_{i}\left(\vec{x}-\vec{x}_{i}\right)=\bar{u}_{i}+S\left(\vec{x}-\vec{x}_{i}\right)+H\left(\vec{x}-\vec{x}_{i}\right)
$$

Therefore, analogous to the second-order case, no new extrema will be formed if

$$
\delta u_{i}^{\min } \leq S\left(\vec{x}-\vec{x}_{i}\right)+H\left(\vec{x}-\vec{x}_{i}\right) \leq \delta u_{i}^{\max }
$$

As in the work of Barth, ${ }^{9}$ the limited form of the higher-order accurate reconstruction can be expressed as

$$
R_{i}\left(\vec{x}-\vec{x}_{i}\right)=\bar{u}_{i}+\phi_{i}\left(S\left(\vec{x}-\vec{x}_{i}\right)+H_{i}\left(\vec{x}-\vec{x}_{i}\right)\right)
$$

Given this formulation, the same limiting procedure used in Section III can be applied to the higher-order reconstruction.

Some previous works ${ }^{5,12}$ have suggested a formulation where the limiter value multiplies only the secondorder terms while the higher-order terms are "switched off" when discontinuities are detected. This formulation has the form of

$$
R_{i}\left(\vec{x}-\vec{x}_{i}\right)=\bar{u}_{i}+\left(\phi_{i}\left(1-\sigma_{i}\right)+\sigma_{i}\right) S\left(\vec{x}-\vec{x}_{i}\right)+\sigma_{i} H\left(\vec{x}-\vec{x}_{i}\right)
$$

where $\sigma_{i}$, the discontinuity detector, is zero near discontinuities and one in smooth regions of the flow. However, this approach may violate the monotonicity requirement as the higher-order terms may have contributed to reducing the overshoot in the unlimited reconstruction used in determining the value of $\phi$. Therefore, the value of $\phi$ computed may be insufficient to reduce the slope such that no overshoots occur when the higher-order reconstruction terms are disabled. The additional parameters introduced in a smooth switching function for $\sigma_{i}$ can be considered a further disadvantage. 


\section{IV.B. Accuracy}

For the second-order case, a limiter provides sufficient accuracy in smooth regions as long as $|\phi-1| \leq \mathcal{O}(\Delta x)$ since this results in an error that is on the order of the quadratic term in the Taylor expansion. However, when a $3^{\text {rd }}$ and $4^{\text {th }}$ order scheme is used, the limiter must satisfy $|\phi-1| \leq \mathcal{O}\left(\Delta x^{2}\right)$ and $|\phi-1| \leq \mathcal{O}\left(\Delta x^{3}\right)$, respectively, for the effect of the limiter in smooth regions to be on the order of truncation error.

For this reason, Venkatakrishnan's limiter will not provide sufficient accuracy even in smooth regions without any local extrema. While the Barth-Jespersen limiter does satisfy these conditions, the lack of differentiability will make achieving a steady-state solution difficult. Therefore, we seek a new approximation for $\min (1, y)$ for use in the limiting procedure that will be monotone and differentiable, and also satisfy $|\widetilde{\min }(1, y)-1| \leq \mathcal{O}\left(\Delta x^{3}\right)$ for $y \geq y_{t}$. For this function, we propose the form

$$
\widetilde{\min }(1, y)=\left\{\begin{array}{cc}
P(y) & y<y_{t} \\
1 & y \geq y_{t}
\end{array}\right.
$$

where $1<y_{t}<2$ is a threshold and $P(y)$ is the cubic polynomial satisfying

$$
\begin{array}{cc}
\left.P\right|_{0}=0 & \left.P\right|_{y_{t}}=1 \\
\left.\frac{d P}{d y}\right|_{0}=1 & \left.\frac{d P}{d y}\right|_{y_{t}}=0
\end{array}
$$

The resulting polynomials for $y_{t}=1.5,1.75$ are plotted in Figure 2 . The choice of $y_{t}$ is a compromise between

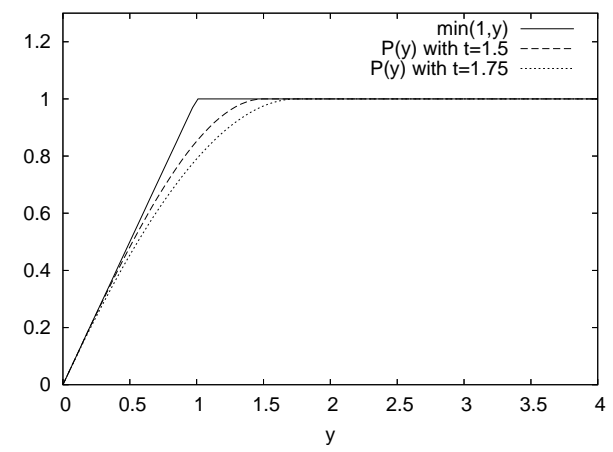

Figure 2. $\widetilde{\min }(1, y)$ with $y_{t}=1.5$ and $y_{t}=1.75$ compared to $\min (1, y)$.

maintaining accuracy in near-extrema (which requires that $y_{t}$ be strictly less than two) and maintaining good convergence properties (which is easier with larger values of $y_{t}$ ). For the results presented in this work, we use $y_{t}=1.5$.

\section{IV.C. Uniform Regions}

In regions of flow which are nearly uniform, extrema will occur frequently in the transient solution. Therefore, it is desirable to eliminate the effect of the limiter in those regions. This can be achieved by "switching off" the limiter when

$$
\delta u \equiv\left(\delta u_{i}^{\max }-\delta u_{i}^{\min }\right)<(K \Delta x)^{\frac{3}{2}}
$$

To maintain differentiability, the following procedure is proposed :

$$
\phi_{i}=\sigma_{i}+\left(1-\sigma_{i}\right) \widetilde{\phi}_{i}
$$

where $\widetilde{\phi}_{i}$ is the limiter value as calculated by the procedure in Section III and $\sigma$ is the following function:

$$
\sigma_{i}=\left\{\begin{array}{cc}
1 & \delta u^{2} \leq(K \Delta x)^{3} \\
s\left(\frac{\delta u^{2}-(K \Delta x)^{3}}{(K \Delta x)^{3}}\right) & (K \Delta x)^{3}<\delta u^{2}<2(K \Delta x)^{3} \\
0 & \delta u^{2} \geq 2(K \Delta x)^{3}
\end{array}\right.
$$


where the transition function $s$ is defined by

$$
s(y)=2 y^{3}-3 y^{2}+1
$$

and is plotted in Figure 3.

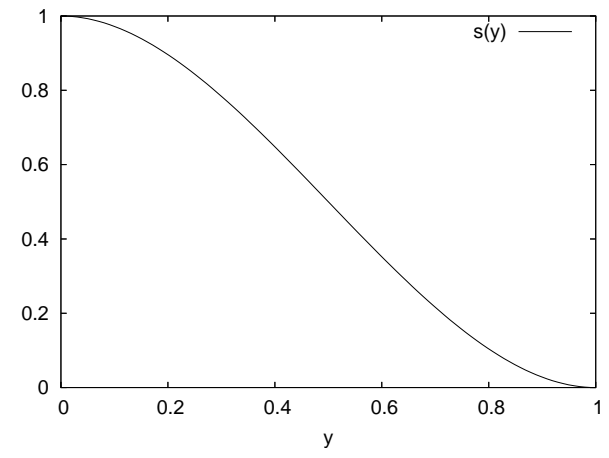

Figure 3. Transition function $s(y)=2 y^{3}-3 y^{2}+1$ used to smoothly disable the limiter in nearly uniform regions.

An added benefit of this approach is that control volumes with $\sigma_{i}=1$ necessarily have $\phi_{i} \equiv 1$. Therefore, the reconstruction procedure is sped up, since $\widetilde{\phi_{i j}}$ need not be evaluated at each Gauss point in uniform flow regions.

\section{Results}

The presented results were obtained using a Newton-GMRES ${ }^{22}$ vertex-centered finite-volume solver. The solution process consists of two stages. In the preiteration stage the linear system resulting from a localtimestepping is solved at each iteration. The Jacobian from the first-order accurate scheme is used on the lefthand-side and the full-order accurate flux is used on the right-hand-side. At each Newton iteration, the linear system is approximately solved using incomplete-lower-upper factorization (ILU) preconditioned GMRES. During the second stage, the left-hand-side is replaced with the full-order accurate Jacobian as described in our previous work. ${ }^{21}$ In the subsonic and transonic cases, the local timestepping is also eliminated at this stage allowing the solution to converge quadratically. Due to the highly non-linear nature of the supersonic case, local time-stepping continues to be used during the second-stage.

In addition to results using the new limiter, results are also presented for the high-order scheme using the procedure in Section IV.A but with Venkatakrishnan's limiting function. For Venkatakrishnan's function we use a tuning parameter of $K=2$ and for the new limiter we use $K=1$.

\section{V.A. Subsonic Flow}

The first test case presented aims to demonstrate the effects of limiters in shockless regions of the flow. For this purpose, we consider subsonic flow over a NACA 0012 airfoil at Mach 0.3. The computational mesh consists of 4656 control-volumes and is shown in Figure 4. We will consider second- and fourth-order schemes using Venkatakrishnan's limiter, the new limiter, and no limiter. The application of limiters to this flow is expected to create additional dissipation due to the clipping of extrema and near-extrema. The effects of this dissipation can best be visualized by plotting the entropy of the flow. Since the flow is inviscid and subsonic, we expect entropy to remain constant.

Plots of the entropy near the leading edge of the airfoil are shown in Figure 5; all plots use the same logarithmic scale. From these plots, we can make a number of observations. Not surprisingly, the fourth-order schemes outperform the second-order schemes when the same limiter is used. Also, the new limiter results in lower entropy production for both the second-order scheme and the fourth-order scheme compared to the results using Venkatakrishnan's limiter. The benefit of using the new limiter is particularly significant for the fourth-order scheme. In fact, the fourth-order scheme using the new limiter outperforms the second-order scheme with no limiter. The source of error for these two cases is different. The entropy produced in the limited fourth-order scheme comes primarily from the clipping of the maximum at the stagnation point. The 


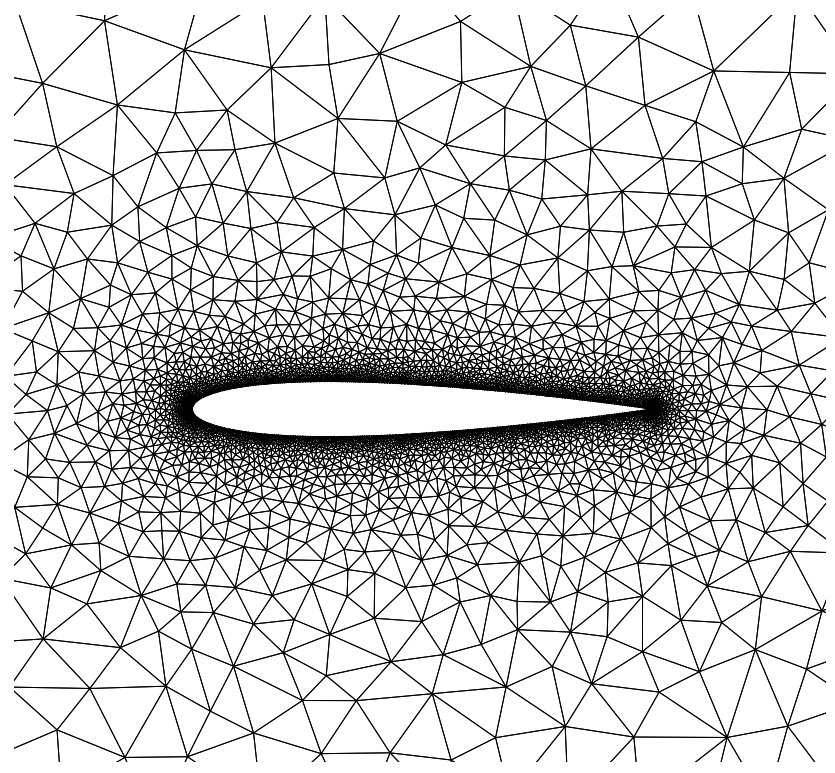

Figure 4. Computational mesh consisting of 4656 control volumes.

second-order unlimited scheme, on the other hand, shows entropy production which is more homogeneously distributed along the airfoil.

The convergence plots are shown in Figure 6 and total computation time ${ }^{\mathrm{a}}$ is given in Table 1. Preiterations were carried out until a relative residual drop of $10^{-3}$ was obtained before switching to a Newton scheme. Using this convergence strategy the application of limiters was actually beneficial to convergence for the fourth-order scheme. A possible reason for this is that the additional dissipation introduced by the limiter gives the scheme some of the properties of a lower-order discretization which is known to have better convergence. This also helps explain why the more dissipative Venkatakrishnan limiter converges faster than the new limiter. It should be noted however that when using the unlimited schemes faster convergence can be obtained by switching to the Newton stage after fewer preiterations (for example after a residual drop of $10^{-2}$ ). This strategy does not work in the limited cases due to the additional non-linearity introduced by the limiter.

\begin{tabular}{|c|c|c|}
\hline Order & Limiter & Computational Time \\
\hline \hline 2nd & None & $10.0 \mathrm{~s}$ \\
\hline 2nd & Venkatakrishnan & $10.1 \mathrm{~s}$ \\
\hline 2nd & New & $13.4 \mathrm{~s}$ \\
\hline 4th & None & $22.3 \mathrm{~s}$ \\
\hline 4th & Venkatakrishnan & $21.3 \mathrm{~s}$ \\
\hline 4th & New & $30.1 \mathrm{~s}$ \\
\hline
\end{tabular}

Table 1. Computational time for subsonic test case.

\section{V.B. Transonic Flow}

Next, we present results for transonic flow over a NACA 0012 airfoil at Mach 0.8 and an angle of attack of 1.25 degree. The same mesh is used as in the subsonic case presented in V.A.

The desired property of the limiter having a value of $\phi=1$ in smooth regions is considered in Figure 7. As expected, Barth-Jespersen's approach shows the limiter being activated only where local extrema are expected. This occurs near the strong shock at the top of the airfoil and the weaker shock at the bottom. Although undesirable, it also occurs in various other control volumes adjacent to the airfoil. The new limiter

\footnotetext{
${ }^{\mathrm{a} C}$ Computational time is based on using a single processor core of an Intel Q6600.
} 


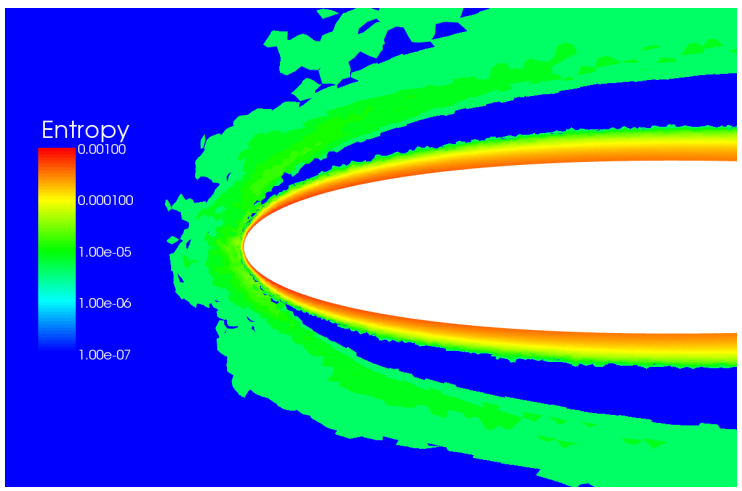

(a) 2nd-order no limiter

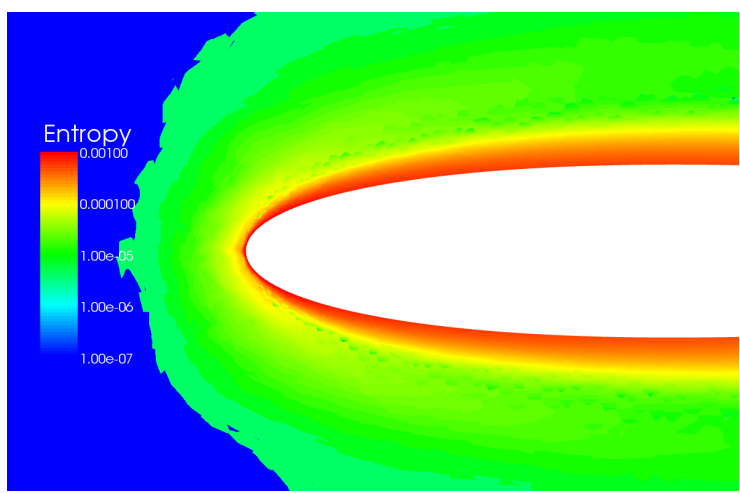

(c) 2nd-order Venkatakrishnan limiter

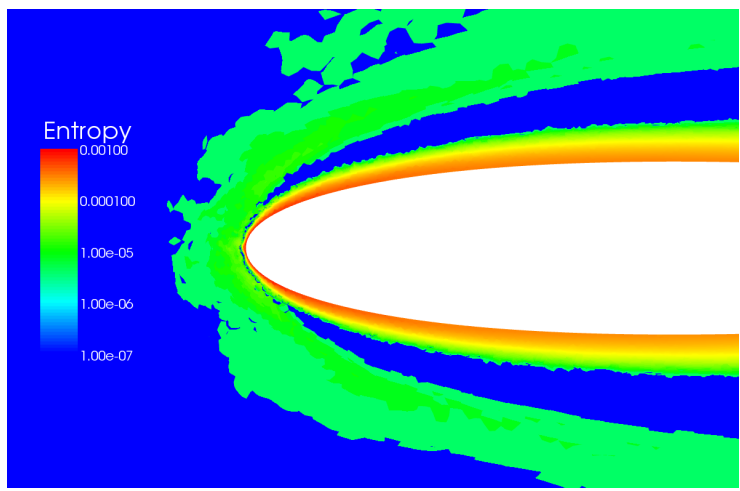

(e) 2nd-order new limiter

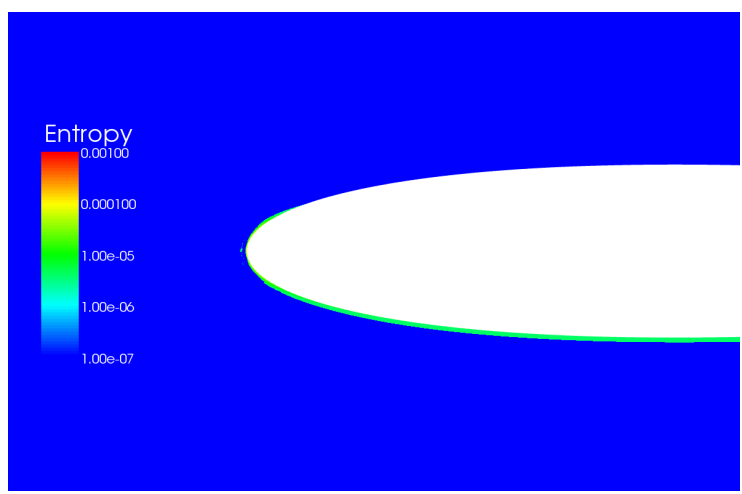

(b) 4th-order no limiter

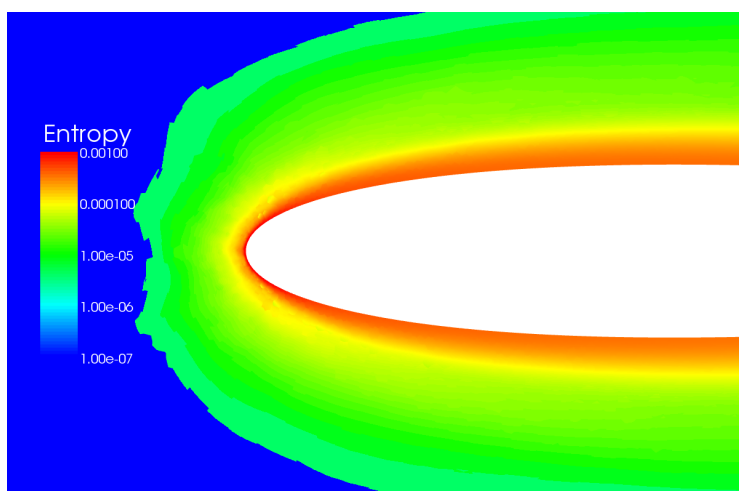

(d) 4th-order Venkatakrishnan limiter

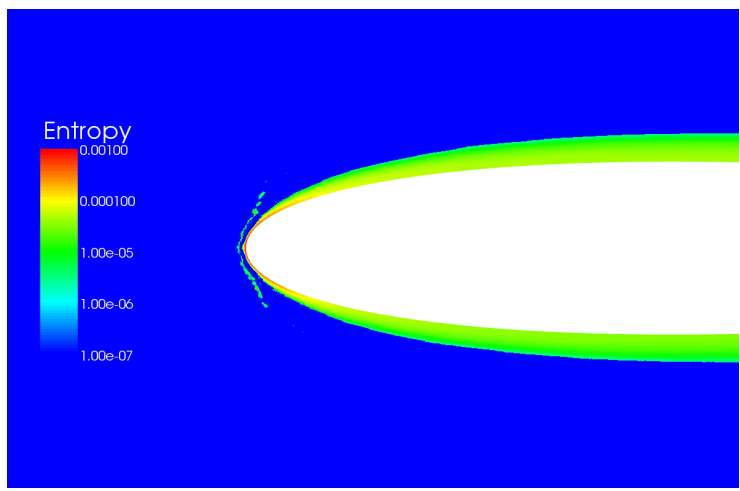

(f) 4th-order new limiter

Figure 5. Difference in dimensionless entropy from the freestream value for Mach 0.3 flow around an airfoil. All plots use the same logarithmic scale. 


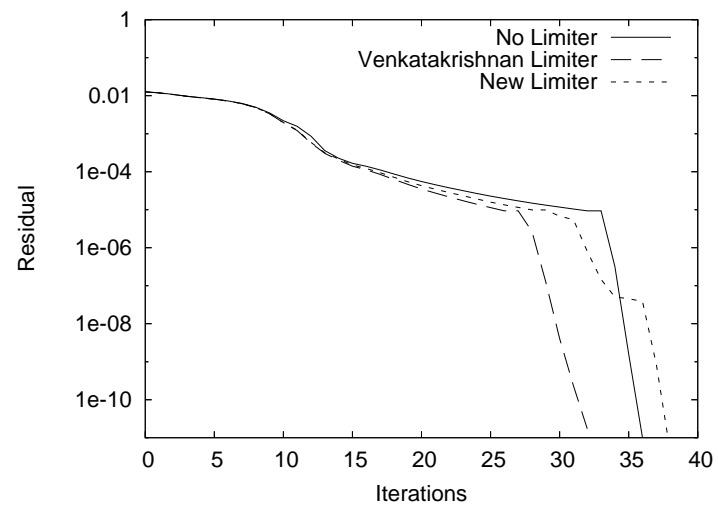

(a) 2nd-order

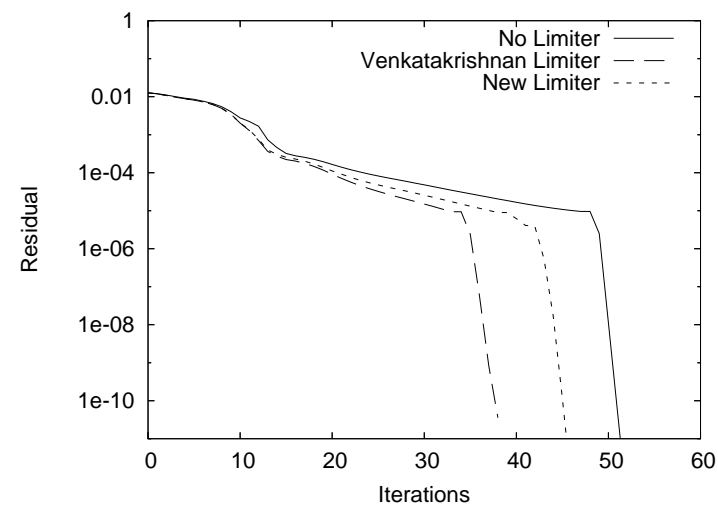

(b) 4th-order

Figure 6. Convergence plots for subsonic flow around an airfoil.

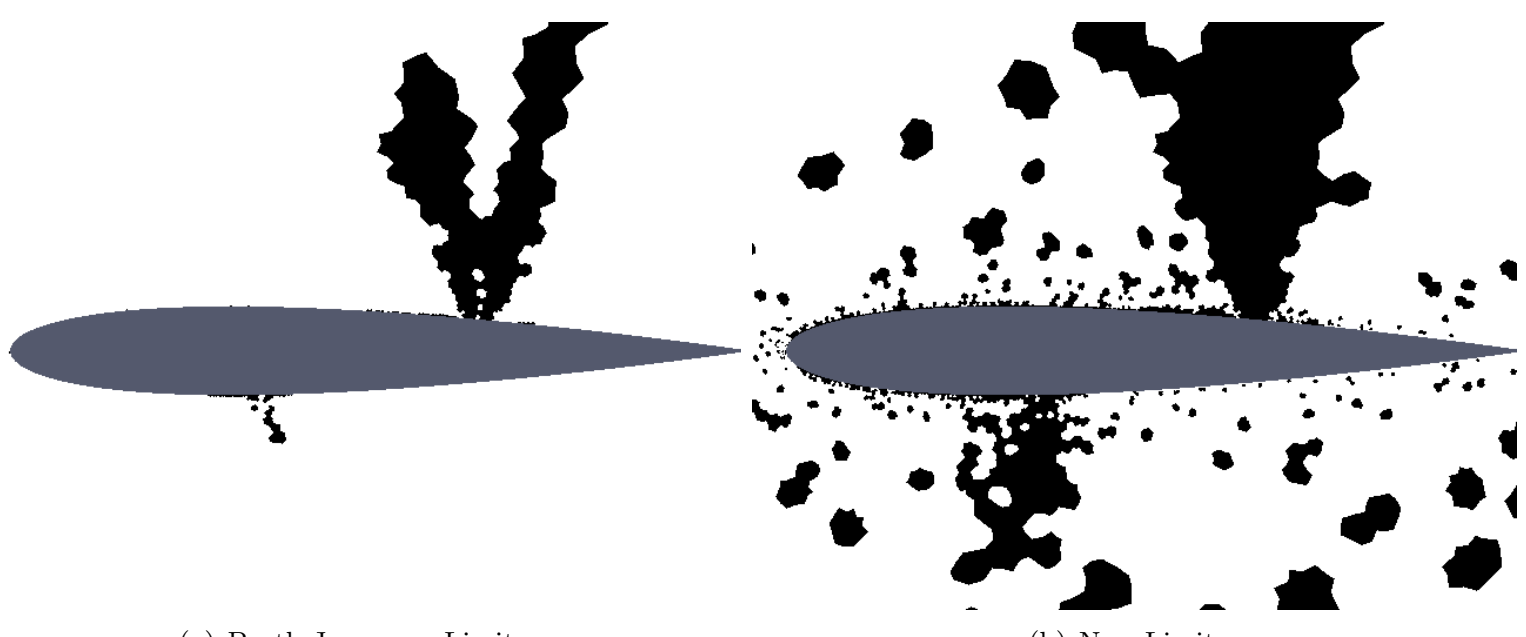

(a) Barth-Jespersen Limiter

(b) New Limiter

Figure 7. Control volumes where the limiter value is $\phi<1$ for any of the unknowns are shown in black. 
is active in a slightly broader area but remains inactive in the majority of control-volumes. No plot is shown for Venkatakrishnan's limiter as it has a value of $\phi<1$ in all control-volumes.

Next, we consider the convergence properties of the new limiting scheme. Figure 8, shows the convergence of the scheme with the new limiter relative to Venkatakrishnan's limiter for second and fourth order accurate schemes. We have also provided the computational time required for convergence in Table 2. As in the

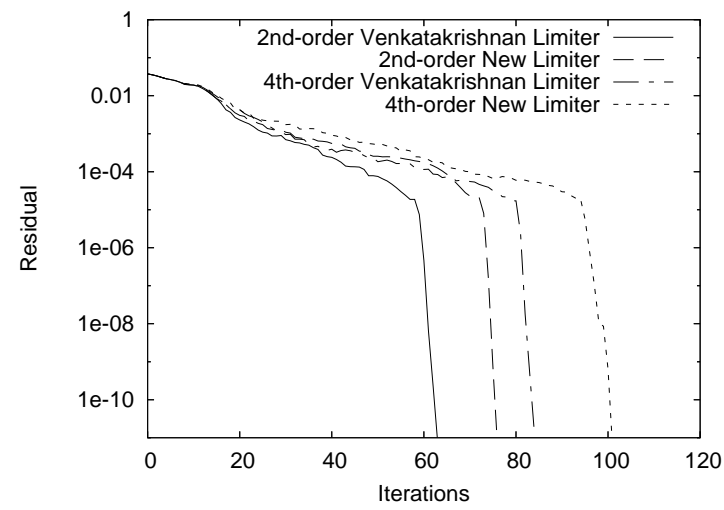

Figure 8. Convergence history for transonic case.

\begin{tabular}{|c|c|c|}
\hline Order & Limiter & Computational Time \\
\hline \hline 2nd & Venkatakrishnan & $17.7 \mathrm{~s}$ \\
\hline 2nd & New & $22.8 \mathrm{~s}$ \\
\hline 4th & Venkatakrishnan & $40.7 \mathrm{~s}$ \\
\hline 4th & New & $52.8 \mathrm{~s}$ \\
\hline
\end{tabular}

Table 2. Computational time for transonic test case.

subsonic case, Venkatakrishnan's limiter converges faster than the new limiter and the second-order scheme converges faster than the fourth-order scheme.

Assessing the quality of the solution is somewhat difficult in this case as the results are tightly clustered together. Therefore, we only present the pressure plot along the airfoil produced by the second-order Venkatakrishnan limiter compared to the fourth-order approach with the new limiter in Figure 9. The result demonstrates that both schemes are effective at eliminating the oscillation near the shocks. The lower diffusivity of the new limiter combined with the fourth-order accurate scheme gives a qualitatively superior result. In particular, the weak shock on the lower surface of the airfoil is significantly better defined than in the second-order solution.

In Figure 10, we demonstrate the effect of using an approach of "switching-off" the high-order terms as in other works. ${ }^{5,12}$ We use Equation 6, which detects uniform flow to switch on the high-order terms only in uniform flow regions. As discussed theoretically in Section IV.A, the results demonstrate that this method violates monotonicity in certain regions.

\section{V.C. Supersonic Flow}

The next test case consists of supersonic flow in a duct with a 12.5 degree ramp. The flow consists of interacting shocks and expansion waves as seen in Figure 11. The mesh consisting of 2322 control-volumes is shown in Figure 12. For this test case we will consider Venkatakrishnan's limiter and the new limiter applied to both second- and fourth-order schemes. Convergence history is presented in Figure 13 and computational time is given in Table 3. Consistent with the other test-cases, the fourth-order scheme requires more iterations to converge. Although Venkatakrishnan's limiter exhibits better convergence for the second-order scheme, for the fourth-order scheme the opposite is true.

To simplify the analysis of the solution, we will consider the pressure plot along a line in the freestream direction that is located at $\frac{1}{3}$ of the distance from the lower to the upper wall. The four computed results, along with a second-order result from a much finer mesh (34297 control-volumes) are plotted in Figure 14. 


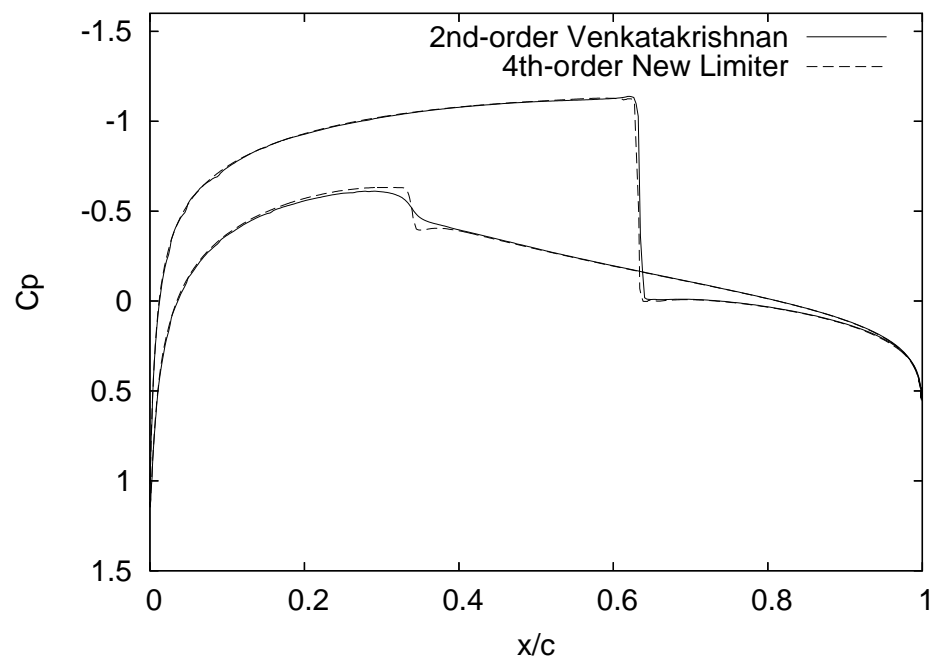

Figure 9. Surface pressure profiles for transonic flow over a NACA0012 airfoil.

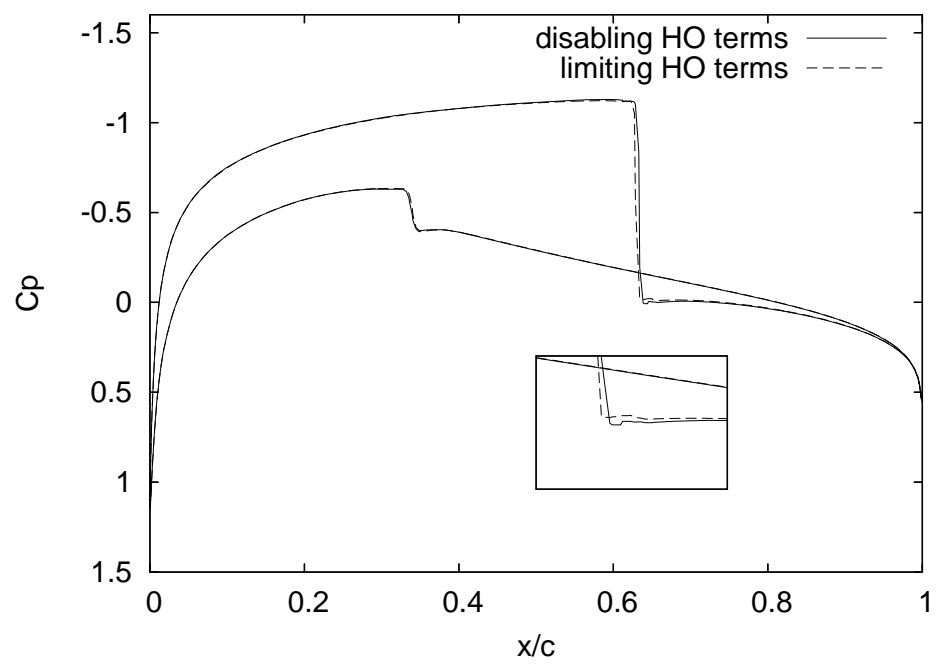

Figure 10. Surface pressure profiles for transonic flow over a NACA0012 airfoil.

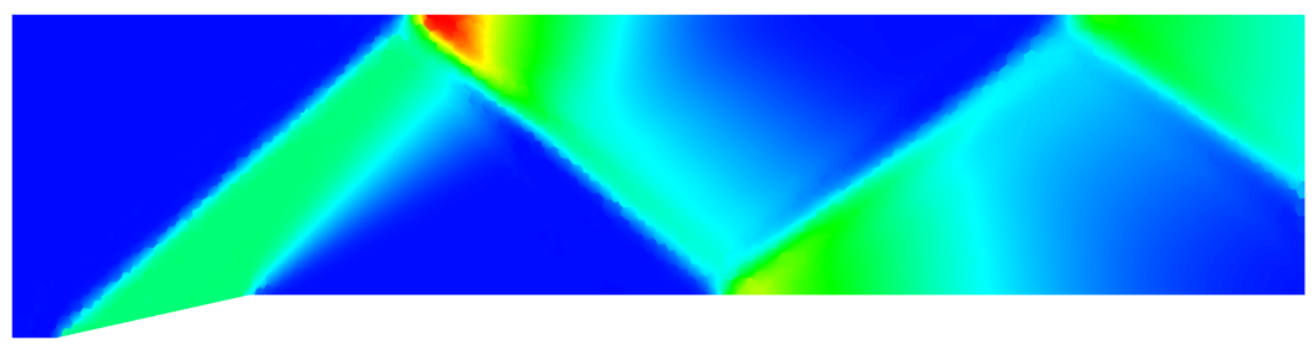

Figure 11. Pressure plot for supersonic flow in a duct

\begin{tabular}{|c|c|c|}
\hline Order & Limiter & Computational Time \\
\hline \hline 2nd & Venkatakrishnan & $5.6 \mathrm{~s}$ \\
\hline 2nd & New & $17.2 \mathrm{~s}$ \\
\hline 4th & Venkatakrishnan & $19.0 \mathrm{~s}$ \\
\hline 4th & New & $24.7 \mathrm{~s}$ \\
\hline
\end{tabular}

Table 3. Computational time for supersonic test case. 


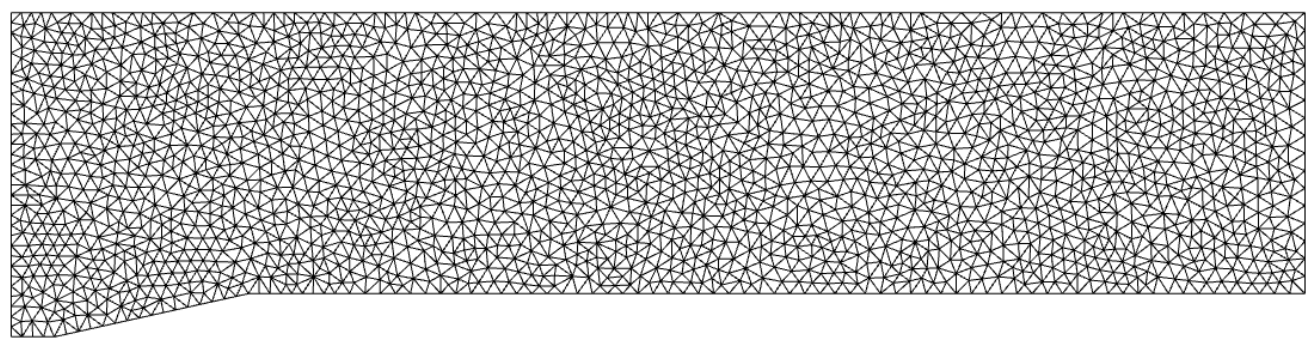

Figure 12. 2322 control-volume mesh used for supersonic test case

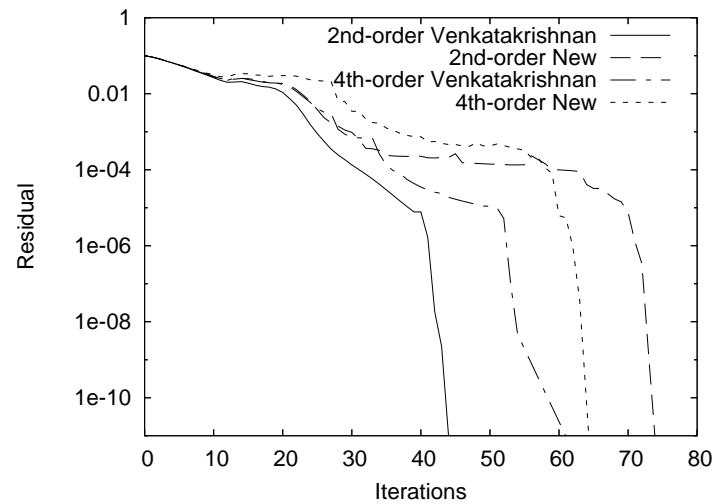

Figure 13. Convergence history for supersonic test case.

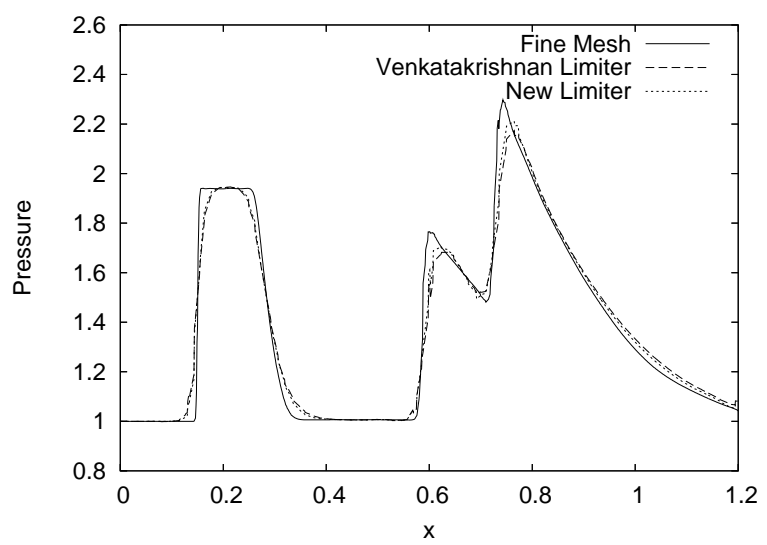

(a) 2nd-order

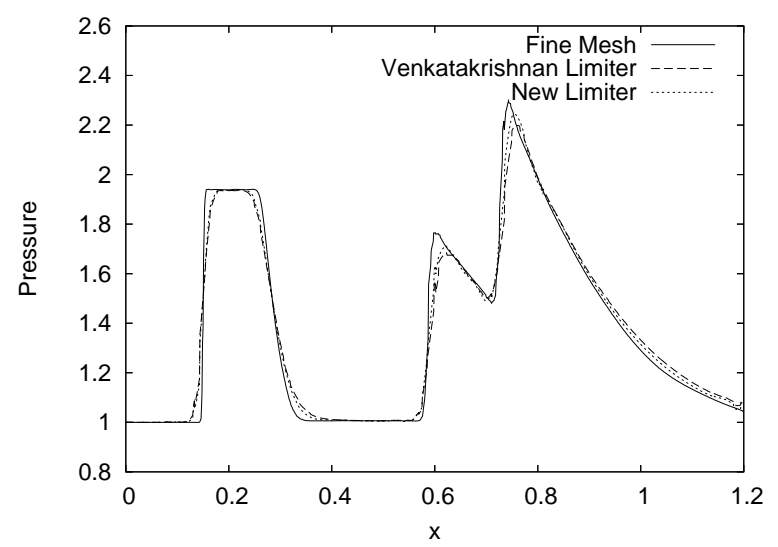

(b) 4th-order

Figure 14. Pressure coefficient plot along $y=\frac{1}{3}$ for supersonic duct. 
We note that all the schemes are effective at preventing oscillations at the shocks. Considering the finemesh result as being "exact" we can easily rank the quality of the coarse mesh solutions. In particular, by observing the peak pressure, we can establish that the fourth-order schemes outperform the second-order schemes. We also see that the new limiter outperforms Venkatakrishnan's. In fact, based on the peak pressure, the new limiter used with a second-order scheme is somewhat superior to the fourth-order schemes with Venkatakrishnan's limiter. It is also notable that the fourth-order schemes produce the desired "square" shape following the initial shock (before any interaction with expansion waves occurs). The second-order schemes produce a rounder and more diffusive solution.

\section{Conclusion}

A limiting procedure for higher-order accurate unstructured finite-volume methods has been proposed. This scheme demonstrates good convergence properties and maintains high-order accuracy in smooth regions were no extrema exist. Since the reconstruction stencil and least-squares matrices do not change at each iteration, the reconstruction procedure is much faster than that of ENO schemes. The drawback of our method is that "clipping" of naturally occurring smooth extrema does occur. However our numerical results show that this does not impede our approach from obtaining a solution that is qualitatively superior to the second-order method for a variety of flow regimes. Furthermore, the new limiter can also be applied to secondorder schemes. In this case, it demonstrates significantly lower dissipation without violating monotonicity at the cost of slightly poorer convergence properties when compared to Venkatakrishnan's approach.

\section{Acknowledgments}

This work was supported by the Canadian Natural Sciences and Engineering Research Council under Grant OPG-0194467.

\section{References}

\footnotetext{
${ }^{1}$ Zingg, D., De Rango, S., Nemec, M., and Pulliam, T., "Comparison of Several Spatial Discretizations for the Navier-Stokes Equations," J. Comp. Phys., Vol. 160, 2000, pp. 683-704.

${ }^{2}$ De Rango, S. and Zingg, D. W., "Higher-order spatial discretization for turbulent aerodynamic computations," AIAA J., Vol. 39, No. 7, July 2001, pp. 1296-1304.

${ }^{3}$ Barth, T. J. and Frederickson, P. O., "Higher Order Solution of the Euler Equations on Unstructured Grids Using Quadratic Reconstruction," AIAA paper 90-0013, Jan. 1990.

${ }^{4}$ Barth, T. J., "Aspects of Unstructured Grids and Finite-Volume Solvers for the Euler and Navier-Stokes Equations," Lecture Series 1994-05, von Karman Institute for Fluid Dynamics, Rhode-Saint-Genèse, Belgium, March 1994.

${ }^{5}$ Delanaye, M. and Essers, J. A., "Quadratic-reconstruction finite volume scheme for compressible flows on unstructured adaptive grids," AIAA J., Vol. 35, No. 4, April 1997, pp. 631-639.

${ }^{6}$ van Leer, B., "Towards the Ultimate Conservative Difference Scheme. V. A Second-Order Sequel to Godunov's Method," J. Comp. Phys., Vol. 32, 1979, pp. 101-136.

${ }^{7}$ Barth, T. J. and Jespersen, D. C., "The Design and Application of Upwind Schemes on Unstructured Meshes," AIAA paper 89-0366, Jan. 1989.

${ }^{8}$ Venkatakrishnan, V., "On the Accuracy of Limiters and Convergence to Steady-State Solutions," AIAA paper 93-0880, Jan. 1993.

${ }^{9}$ Barth, T. J., "Recent Developments in High Order K-Exact Reconstruction on Unstructured Meshes," AIAA paper 93-0668, Jan. 1993.

${ }^{10}$ Ollivier-Gooch, C. F., "High-Order ENO Schemes for Unstructured Meshes Based on Least-Squares Reconstruction," AIAA paper 97-0540, Jan. 1997.

${ }^{11}$ Geuzaine, P., Delanaye, M., and Essers, J.-A., "Computation of High Reynolds Number Flows with an Implicit Quadratic Reconstruction Scheme on Unstructured Grids," Proc. 13th AIAA CFD Conf., Amer. Inst. Aero. Astro., 1997, pp. 610-619.

${ }^{12}$ Nejat, A. and Ollivier-Gooch, C., "A High-Order Accurate Unstructured Newton-Krylov Solver for Inviscid Compressible Flows," 36th AIAA Fluid Dynamics Conference, 2006, AIAA 2006-3711.

${ }^{13}$ Abgrall, R., "On Essentially Non-oscillatory Schemes on Unstructured Meshes: Analysis and Implementation," J. Comp. Phys., Vol. 114, No. 1, 1994, pp. 45-58.

${ }^{14}$ Shu, C.-W., Zang, T. A., Erlebacher, G., Whitaker, D., and Osher, S., "High-order ENO Schemes Applied to Two- and Three-Dimensional Compressible Flow," Appl. Num. Math., Vol. 9, No. 1, 1992, pp. 45-71.

${ }^{15}$ Godfrey, A. G., Mitchell, C. R., and Walters, R. W., "Practical Aspects of Spatially High-Order Accurate Methods," AIAA J., Vol. 31, No. 9, Sept. 1993, pp. 1634-1642.

${ }^{16}$ Friedrich, O., "Weighted Essentially Non-Oscillatory Schemes for the Interpolation of Mean Values on Unstructured Grids," J. Comp. Phys., Vol. 144, No. 1, July 1998, pp. 194-212.
} 
${ }^{17} \mathrm{Hu}$, C. Q. and Shu, C. W., "Weighted essentially non-oscillatory schemes on triangular meshes," J. Comp. Phys., Vol. 150, No. 1, March 1999, pp. 97-127.

${ }^{18}$ Jiang, G.-S. and Shu, C.-W., "Efficient Implementation of Weighted ENO Schemes," J. Comp. Phys., Vol. 126, No. 1, June 1996, pp. 202-228.

${ }^{19}$ Ollivier-Gooch, C. F., "Quasi-ENO Schemes for Unstructured Meshes Based on Unlimited Data-Dependent Least-Squares Reconstruction," J. Comp. Phys., Vol. 133, No. 1, 1997, pp. 6-17.

${ }^{20}$ Ollivier-Gooch, C. F. and Van Altena, M., "A High-order Accurate Unstructured Mesh Finite-Volume Scheme for the Advection-Diffusion Equation," J. Comp. Phys., Vol. 181, No. 2, 2002, pp. 729-752.

${ }^{21}$ Michalak, K. and Ollivier-Gooch, C., "Matrix-Explicit GMRES for a Higher-Order Accurate Inviscid Compressible Flow Solver," Proc. 18th AIAA CFD Conf., 2007.

${ }^{22}$ Saad, Y. and Schultz, M. H., "GMRES: A Generalized Minimal Residual Algorithm for Solving Nonsymmetric Linear Systems," SIAM J. Sci. Stat. Comp., Vol. 7, No. 3, July 1986, pp. 856-869. 